\title{
Effect of oxygen and nitrogen functionalization on the physical and electronic structure of graphene
}

\author{
Alexander J. Marsden ${ }^{1}$, Peter Brommer ${ }^{1,2}$, James J. Mudd', M. Adam Dyson ${ }^{1}$, Robert Cook ${ }^{1}$, María Asensio ${ }^{3}$, \\ Jose Avila ${ }^{3}$, Ana Levy ${ }^{3}$, Jeremy Sloan ${ }^{1}$, David Quigley ${ }^{1,2}$, Gavin R. Bell ${ }^{1}$, and Neil R. Wilson ${ }^{1}(\varangle)$ \\ ${ }^{1}$ Department of Physics, University of Warwick, Coventry, UK \\ ${ }^{2}$ Centre for Scientific Computing, University of Warwick, Coventry, UK \\ ${ }^{3}$ Synchrotron SOLEIL, L'Orme des Merisiers, Saint Aubin-BP 48, 91192 Gif sur Yvette Cedex, France
}

Received: 7 January 2015

Revised: 20 February 2015

Accepted: 16 March 2015

(C) Neil R. Wilson 2015. This article is published with open access at link.springer.com

\section{KEYWORDS}

graphene,

functionalization, chemical vapor deposition, density functional theory

\begin{abstract}
Covalent functionalization of graphene offers opportunities for tailoring its properties and is an unavoidable consequence of some graphene synthesis techniques. However, the changes induced by the functionalization are not well understood. By using atomic sources to control the extent of the oxygen and nitrogen functionalization, we studied the evolution in the structure and properties at the atomic scale. Atomic oxygen reversibly introduces epoxide groups whilst, under similar conditions, atomic nitrogen irreversibly creates diverse functionalities including substitutional, pyridinic, and pyrrolic nitrogen. Atomic oxygen leaves the Fermi energy at the Dirac point (i.e., undoped), whilst atomic nitrogen results in a net n-doping; however, the experimental results are consistent with the dominant electronic effect for both being a transition from delocalized to localized states, and hence the loss of the signature electronic structure of graphene.
\end{abstract}

\section{Introduction}

Pristine graphene, with a perfect single atom thick hexagonal carbon lattice, has many remarkable properties. However, many of the diverse routes currently pursued for large scale production generate graphene sheets that are no longer pristine, but rather in which functional groups are covalently attached to the carbon lattice either intentionally, as part of the synthesis procedure, as is the case of graphene oxide [1], or inadvertently, as can often happen in electrochemical exfoliation [2]. At the same time, covalent functionali- zation is actively being pursued to tailor the properties of graphene towards certain applications. For example, as-produced graphene oxide is a highly soluble material that is much easier to process than pristine graphene [3], whilst nitrogen functionalization, which has been shown to dramatically increase the electrocatalytic activity of graphene [4-8], is being explored as a means to substitutionally dope graphene $[9,10]$.

It is clear that the functionalization of graphene can enhance its functional properties. However, the effect of the covalent functionalization on the physical, chemical, and electronic structure of graphene, and

Address correspondence to Neil.Wilson@warwick.ac.uk 
hence on its properties, is not yet well understood. To some extent, the lack of fundamental understanding of the structure and properties can be explained by the approaches used. Most works on the covalent functionalization of graphene have concentrated on wet chemical approaches because of their low cost and high flexibility. Unfortunately, the resultant materials are complex and only coarse control over their stoichiometry is achievable, complicating fundamental studies of their properties.

A greater control can be gained through gas phase reactions, for example through the oxidation of graphene either by ozone [11, 12], oxygen plasma [13], or under ultra-high vacuum (UHV) conditions by atomic oxygen [14,15]. Atomic oxygen is readily formed by cracking the diatomic oxygen molecule under UHV conditions, either thermally or using a radio-frequency plasma, creating the highly reactive monoatomic oxygen radical. The functionalization by atomic sources offers easy control over the extent of the reaction (the number of functional groups per unit area) through simply varying the exposure time, without the damage due to high energy species that is often induced by plasma sources.

Here, we used chemical vapor deposition (CVD) grown graphene as a model system, and investigated the effects of atomic oxygen and atomic nitrogen in the controllable covalent functionalization of graphene. We compared the changes in chemical composition (measured by X-ray photoemission spectroscopy, XPS), physical structure (measured by aberration corrected transmission electron microscopy, acTEM), and electronic structure (measured by angle resolved photoemission spectroscopy, ARPES) with density functional theory (DFT) modeling. We show that even low levels of functionalization can dramatically change the properties of graphene, and that the effects vary radically depending on the type of functionalization, even when similar experimental conditions are used.

\section{Results and discussion}

\subsection{CVD grown graphene on copper}

Graphene was grown on copper foils by low pressure
CVD (LP-CVD). Scanning electron microscopy (SEM), Raman, and TEM investigations (see electronic supplementary material, ESM, Fig. S1) show an almost complete coverage of predominantly monolayer graphene with few defects and that, under the growth conditions used here, the typical graphene grain size is a few micrometers compared to the typical copper grain size after growth of $\sim 1 \mathrm{~mm}$. After growth, the copper surface becomes faceted underneath the graphene layer, with the surface of each facet atomically smooth $[16,17]$. This enables the use of ARPES to directly study the electronic structure of graphene, whilst the ability to remove the graphene layer from the copper foil by etching away the copper allows the physical structure to be analyzed at atomic resolution by TEM.

ARPES of pristine graphene on copper shows the well-known Dirac cones meeting at the Fermi level. Figures 1(a) and 1(b) show representative ARPES results taken on LP-CVD grown graphene on a copper foil sample, with schematics of the experimental geometry and expected results given in Fig. 1(c). The ARPES measurement here averages over a $\sim 100-\mu$ m diameter region of the sample, which corresponds to an area within one copper grain, but integrated over a large number $(\sim 1,000)$ of graphene grains. However, owing to the preferential orientation of the graphene grains, [17] two sets of Dirac cones are clearly resolved, marked in red and blue. In a slice through the Dirac cones, Fig. 1(b), the linear band dispersion is apparent, meeting at the Fermi level at the Dirac point, i.e., graphene on copper is undoped and of high electronic quality. This result is consistent with previous reports for graphene grown on this type of copper foil under similar conditions, showing that graphene is essentially decoupled from the copper surface $[17,18]$.

Graphene can be transferred from the copper foil, and suspended on a TEM grid to resolve its atomic structure. A high resolution TEM image of the asgrown graphene is shown in Fig. 2. The expected hexagonal graphene lattice is clearly evident (see also the inset fast Fourier transform). The amorphous areas highlighted in blue are residues from the transfer process lying on top of the graphene sheet. Reproducibly, such images show a pristine graphene lattice with no evidence for the presence of defects. 

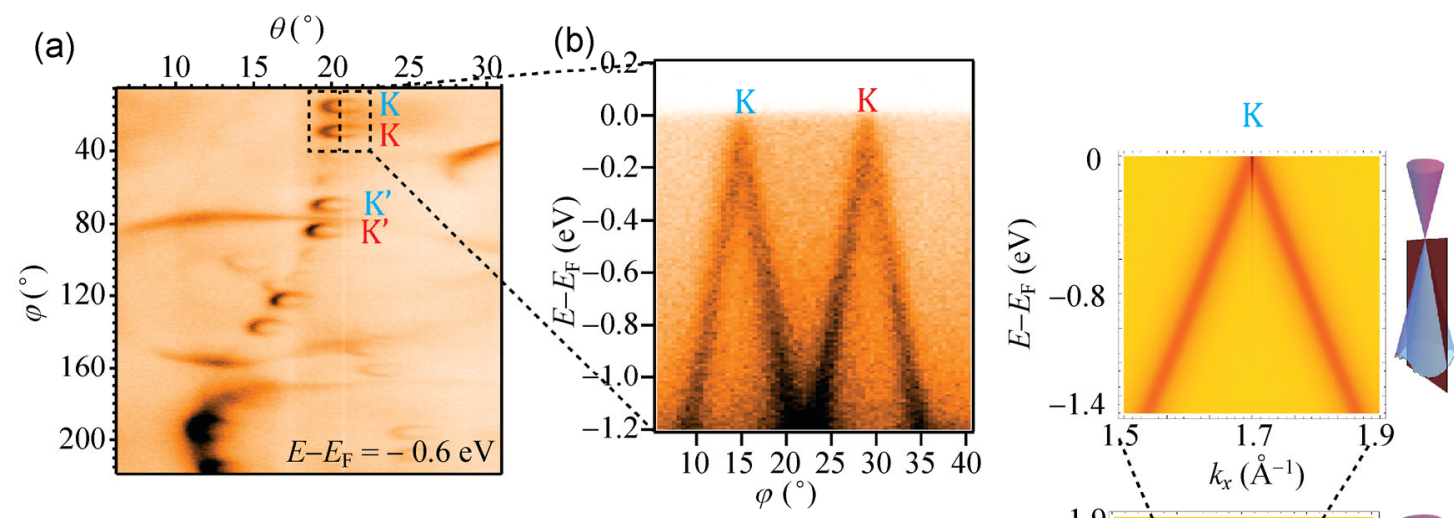

(c)

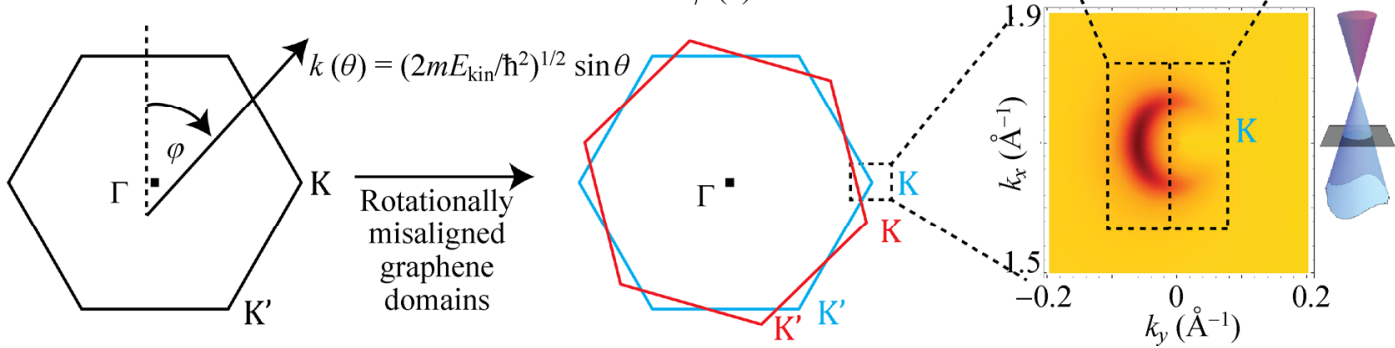

Figure 1 Angle resolved photoemission spectroscopy (ARPES) revealing the electronic structure of polycrystalline graphene on copper foil. (a) Experimental results showing the ARPES intensity as a function of the collection angle at an energy of $0.6 \mathrm{eV}$ below the Fermi level and (b) slices through the Dirac cones (as marked) showing them to be undoped with a linear dispersion around the Fermi energy. Two preferred orientations of graphene are found, with $\mathrm{K}$ points labeled in blue and red. The schematic in (c) shows the relationship between the measurement geometry $(\theta, \varphi)$ and the Brillouin zone of graphene along with simulations of slices through an undoped graphene Dirac cone at constant energy and perpendicular to the $\Gamma-\mathrm{K}$ direction.

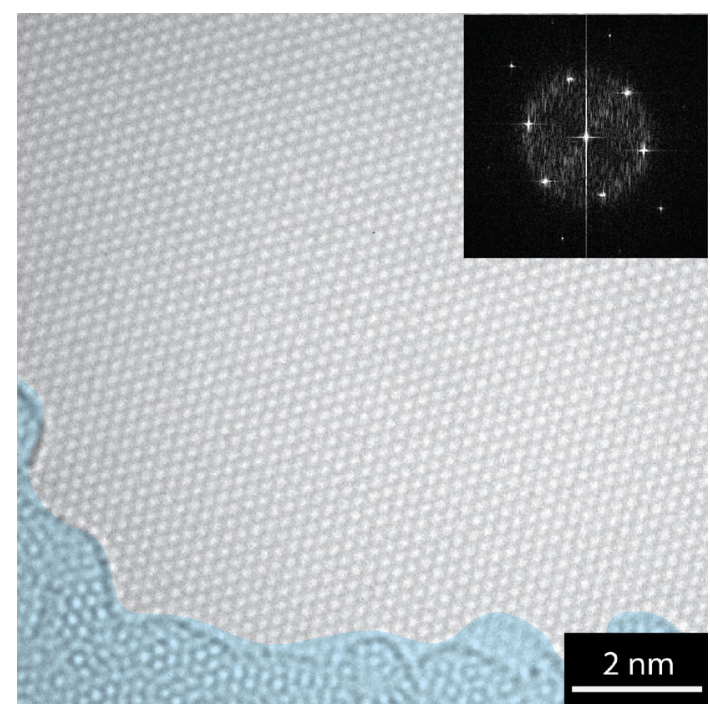

Figure 2 High-resolution transmission electron microscopy image of graphene after removal from the copper foil substrate. The polymer residue is highlighted in blue. (Inset) Fast Fourier transform of the image.

\subsection{Effect of atomic oxygen on the physical and chemical structure}

Graphene on copper samples were exposed to controlled doses of atomic oxygen under UHV conditions (see Methods and Supporting Information for further details). The atomic oxygen source produces a mixture of atomic and molecular oxygen; however, pure $\mathrm{O}_{2}$ was found not to alter the chemical or electronic structure of graphene on copper at exposure levels comparable to the atomic dosing experiments.

Figure 3 shows the XPS spectra of graphene on copper before (pristine) and after exposure to atomic oxygen. The dosing was consecutive and the labeled dose is the total amount at each point (i.e., the actual doses were $10 \mathrm{~s}, 20 \mathrm{~s}$, and $30 \mathrm{~s}$ ). The $\mathrm{C} 1 \mathrm{~s}$ region of the pristine sample, after low temperature annealing, is fit by a single asymmetric Doniach-Sunjic (DS) peak at $284.3 \pm 0.1 \mathrm{eV}$, characteristic of clean graphene [18]. After successive dosing with atomic oxygen, a second peak at $286.0 \pm 0.1 \mathrm{eV}$ appears; this can be assigned to an epoxide (bridge site oxygen adatom) functionality $[14,15]$. The ratio of the area of the two peaks quantifies the percentage of epoxide carbons present; this is plotted as a function of the dose in Fig. 3(b), which shows a roughly linear increase of the concentration

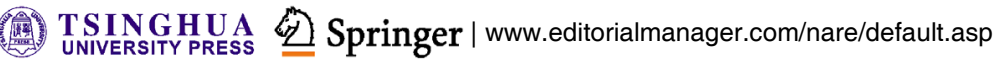


(a)
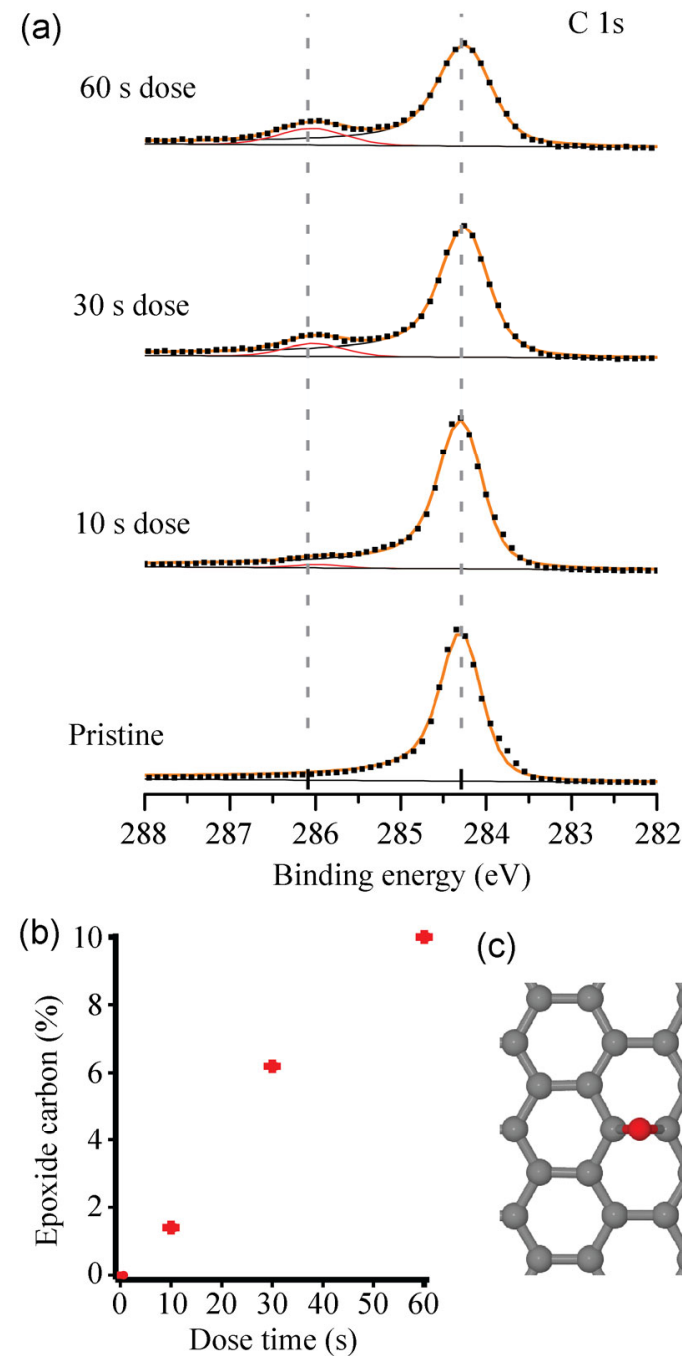

(c)

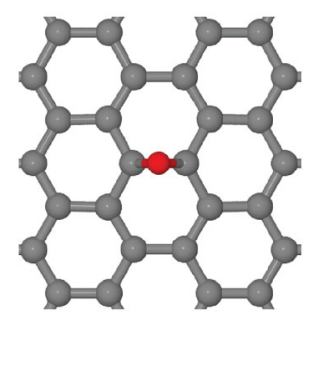

Figure 3 X-ray photoemission spectroscopy (XPS) of graphene on copper exposed to atomic oxygen. (a) XPS of the $\mathrm{C} 1 \mathrm{~s}$ region of graphene before and after exposure to increasing doses of atomic oxygen. The experimental data (black squares) are fit (orange line) with the sum of a Doniach Sunjich peak at $284.3 \pm 0.1 \mathrm{eV}$ (black line) for the graphene-like carbon and a peak at $286.0 \pm 0.1 \mathrm{eV}$ (red line) corresponding to epoxide carbons. (b) Graph of the increase of epoxide carbon percentage with the exposure time to atomic oxygen. (c) Structure of the epoxide group (bridge site oxygen adatom) as predicted by the density functional theory with carbon atoms in grey and oxygen atoms in red.

of the epoxide group with the time. Notably, each epoxide group contains two carbon atoms, thus the atomic percent (at\%) of epoxide oxygen to graphitic carbon (at these low concentrations) is half that amount. Hence, the $60 \mathrm{~s}$ dose resulted in roughly 5 at $\%$ of epoxide oxygen. The geometry of the epoxide group, as calculated by DFT (discussed later), is shown in Fig. 3(c). A detailed analysis of the $\mathrm{O} 1 \mathrm{~s}$ is complicated by the presence of a small amount of oxygen associated with the pristine graphene samples, consistent with previous reports $[18,19]$. However, the O 1s peak increases with the atomic oxygen exposure in a manner consistent with the changes in the $\mathrm{C} 1$ s peak described above (for further information see Figs. S2 and S3 and discussion in the electronic supplementary material). Scanning photoemission microscopy (SPEM) showed that the level of oxygen functionalization was essentially homogeneous across the surface (see Fig. S4).

We found that low levels (up to $\sim 5$ at $\%$ ) of epoxide functionalization could be easily removed by heating at $190{ }^{\circ} \mathrm{C}$ in UHV for $30 \mathrm{~min}$ (see Fig. S5 for XPS), in agreement with Hossain et al. [14]. This is consistent with the oxygen in the epoxide groups being only fairly weakly bound to the graphene sheet. Using realistically large supercells, DFT calculations were performed to investigate the stability of the epoxide groups (see Methods and Supporting Information for further details). The formation energy was calculated as the total energy of the supercell minus the chemical potentials of the atoms referenced to graphene for carbon, and to the atomic species for oxygen and nitrogen. The formation energy for an epoxide group was found to be $-2.0 \mathrm{eV}$, in good agreement with prior work [15]. The calculations also showed that the recombination of two oxygen atoms from two epoxy groups to form $\mathrm{O}_{2}$ and pristine graphene is energetically favorable $(-1.9 \mathrm{eV}$ compared to the two epoxy groups). Annealing could thus result in the removal of the epoxide functionalities, either through the direct desorption of the individual epoxide groups or, more likely, through the diffusion of the epoxide groups until two meet and desorb as the more stable $\mathrm{O}_{2}$ molecule.

For the TEM analysis, graphene was transferred to a TEM support grid and cleaned before exposure under UHV conditions to atomic oxygen at equivalent doses to those shown in Fig. 3. The grids were transferred from the UHV system to an acTEM. In most places a pristine graphene lattice was observed, consistent with the epoxide groups being mobile at room temperature and quickly removed by the electron beam; however, occasional evidence was found for isolated epoxide groups (see Fig. S6). There was no evidence for an increase in the number of 
defects in the graphene lattice or for the presence of other types of oxygen functionality.

\subsection{Effect of atomic nitrogen on the physical and chemical structure}

The evolution of graphene on copper upon exposure to atomic nitrogen under similar conditions (same pressure and power for the cracking source, see Methods) was also studied. The XPS results are presented in Fig. 4, with corresponding survey scans given in Supporting Information (Fig. S7). The C 1s region, Fig. 4(a), is less informative than that observed for atomic oxygen. For the pristine sample, the data is again well fit by a single DS line shape at $284.3 \pm$ $0.1 \mathrm{eV}$. With increasing the exposure to atomic nitrogen, this peak broadens and shifts to slightly higher binding energy, and is no longer well fit by a single DS curve. Instead, in Fig. 4, we fitted the results with an additional broad peak at around $285 \mathrm{eV}$; the width of the peak suggests that it is a composite feature from many different chemical environments, including $\mathrm{C}-\mathrm{N}$ and $\mathrm{C}=\mathrm{N}[20]$.
The corresponding $\mathrm{N} 1 \mathrm{~s}$ region scans are shown in Fig. 4(b). There is no evidence for the presence of nitrogen in the pristine sample. After exposure to atomic nitrogen, there is a clear increase in the intensity in the $\mathrm{N} 1$ s region, which cannot be fit by just one peak. Following the assignments given in previous reports on the CVD growth of nitrogen-doped graphene and nitrogen doping of graphene oxide $[5,6$, $9,21,22]$, we fitted the data with a peak at $400.9 \pm$ $0.1 \mathrm{eV}$ assigned to $\mathrm{N}$ substitutions in the graphene lattice, a peak at $398.7 \pm 0.1 \mathrm{eV}$, which we assigned to both pyrrolic ( $\mathrm{N}$ in a five-membered ring with four $\mathrm{C}$, see Fig. 6(f)) and pyridinic (bonded to two carbon atoms, see Fig. 6(d)) nitrogen. Pyridinic and pyrrolic nitrogen are often assigned independently to peaks at 400 and $399 \mathrm{eV}$, respectively, but the noise levels in the spectra here precluded their independent accurate quantification. Hence, we grouped them into one, vacancy related, peak at $398.7 \mathrm{eV}$. Finally a peak at $396.6 \pm 0.1 \mathrm{eV}$ was assigned to nitrogen adatoms (the nitrogen equivalent of the epoxide group).

As with atomic oxygen, the total amount of
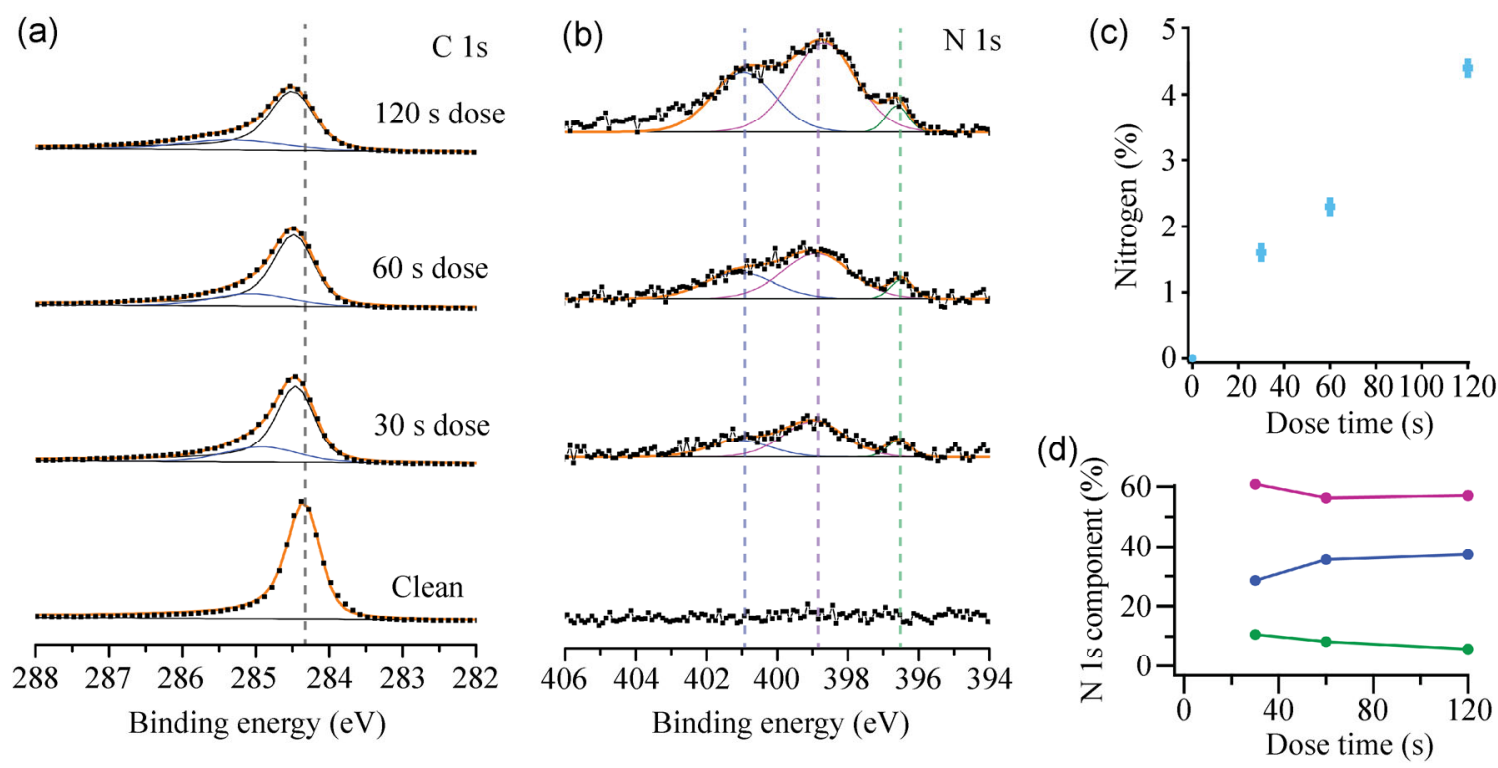

Figure 4 X-ray photoemission spectroscopy (XPS) of graphene on copper exposed to atomic nitrogen. XPS of (a) the C 1s region and (b) the $\mathrm{N} 1 \mathrm{~s}$ region of graphene before and after exposure to increasing doses of atomic nitrogen. In (a), the experimental data (black squares) are fit (orange line) with the sum of a Doniach Sunjich peak at $284.3 \pm 0.1 \mathrm{eV}$ (black line) for the graphene-like carbon and a broad Voigt peak at around $285 \mathrm{eV}$ (blue line) corresponding to multiple chemical environments. In (b), the experimental data (black squares) are fit with a peak at $400.9 \pm 0.1 \mathrm{eV}$ (blue line) assigned to substitutional $\mathrm{N}$, a peak at $398.7 \pm 0.1 \mathrm{eV}$ (purple line) assigned to pyridinic and pyrrolic $\mathrm{N}$, and a peak at $396.6 \pm 0.1 \mathrm{eV}$ (green line) assigned to nitrogen adatoms. (c) Atomic percentage of $\mathrm{N}$ relative to $\mathrm{C}$, calculated from the integrated intensity of the region scans. (d) Percentage of the $\mathrm{N} 1 \mathrm{~s}$ assigned to substitutional (blue), pyridinic/ pyrrolic (purple), and nitrogen adatoms (green). 
functionalization shows a roughly linear increase with increasing the exposure time. This is quantified in Fig. 4(c), which shows the increase in nitrogen content (calculated from the comparison between the $\mathrm{C}$ 1s and N 1s XPS regions for each sample) with the exposure time. The intensity ratios between the individual components of the $\mathrm{N}$ 1s peak are roughly constant over time, Fig.4(d), with substitutional: pyridinic + pyrrolic:adatom approximately in the proportion 30:60:10. After heating at $190{ }^{\circ} \mathrm{C}$ for $12 \mathrm{~h}$, the adatom peak disappeared, but the substitutional and pyridinic/pyrrolic nitrogen remained (see Fig. S8), showing that the atomic nitrogen functionalization was irreversible. After annealing, the proportion of substitutional nitrogen increased significantly relative to the pyridinic/pyrrolic nitrogen, in agreement with the recent results of Scardamaglia et al., who looked at the effect of annealing on nitrogen ion implanted groups in graphene [23].

Thus, the XPS analysis shows a range of chemical environments for nitrogen, but does not provide detailed information on the physical structure. To this end, acTEM was used. Figure 5(a) shows an acTEM image of graphene exposed to a $30 \mathrm{~s}$ dose of atomic nitrogen. As with the pristine graphene, there is some residue from the transfer process. The contrast between the $\mathrm{C}$ and $\mathrm{N}$ atoms is here insufficient to positively identify the sites of the $\mathrm{N}$ functionalization. However, there are clear differences compared with the pristine graphene surface; the most evident is the high concentration of defects. To highlight their position, the image was Fourier filtered to remove the periodicity of the graphene lattice (Fig. 5(b)). Isolated defects can be seen along with clusters of defects (marked in orange). For the isolated defects, two common types are observed. These are shown at higher resolution in Figs. 5(c)-5(f): (d) and (f) are exit wave reconstructions formed from focal series, whilst (c) and (e) are simulations of the corresponding exit wave images starting from the DFT potentials for the optimized geometries (discussed later). (c) and (d) correspond to a divacancy, two missing atoms from the hexagonal lattice, which has reconstructed into 5-7-5-7-5-7 rings [24, 25]; an example of such a structure is labeled in green in (b). (e) and (f) correspond to a mono-vacancy, one atom missing from the hexagonal lattice, examples

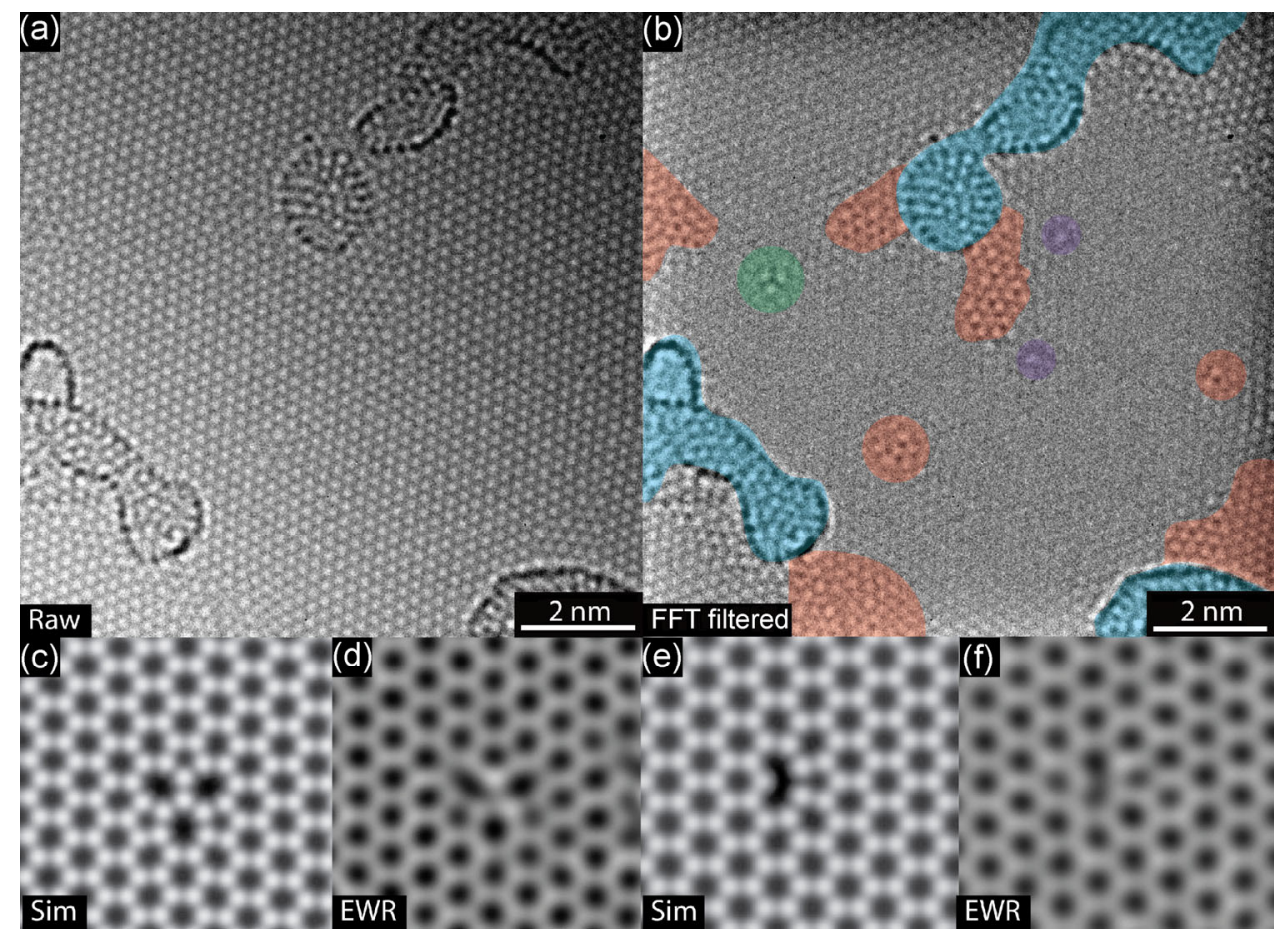

Figure 5 Aberration corrected transmission electron microscopy of graphene exposed to a $30 \mathrm{~s}$ dose of atomic nitrogen before (a) and after (b) Fourier filtering. Different types of defects are highlighted in the Fourier filtered image, as described in the text. Simulations, (c) and (e), and exit wave reconstructions, (d) and (f), of a divacancy and monovacancy, respectively. 
of which are marked in purple in Fig. 5(b). As mentioned earlier, at this resolution, we could not distinguish between $\mathrm{C}$ and $\mathrm{N}$, and thus could not positively identify the position of the nitrogencontaining functional groups. This is evident from the exit wave simulations: (c) was calculated from the structure shown in Fig. 6(f) below, and (e) from Fig. 6(d) (see Methods for details on the simulation). Notably, the clustering of the vacancies results in extended topological defects where the local order is different from that in the graphene lattice, similar to the structures observed in previous high resolution TEM studies of reduced graphene oxide [26]. These extended topological defects are known to induce strain and curvature into the graphene sheet, also increasing the reactivity [27].

For the $30 \mathrm{~s}$ dose of atomic nitrogen, the XPS data suggest that the total $\mathrm{N}$ concentration should be almost 2 at $\%$, of which $~ 1$ at $\%$ is due to a combination of pyridinic and pyrrolic groups. The area of the unit cell in pristine graphene is $0.051 \mathrm{~nm}^{2}$, giving 39 carbon atoms $\mathrm{nm}^{-2}$. A concentration of 2 at $\%$ corresponds to roughly 1 atom $\mathrm{nm}^{-2}$. Thus, the typical spacing between the defects observed here by acTEM (Fig. 5), factoring in the presence of the defect clusters, is consistent with the pyridinic + pyrrolic nitrogen concentration determined by XPS. Interestingly, the TEM analysis shows that these functionalities are associated with varying reconstructions of the lattice due to the vacancies, which are significantly more complicated than the usually considered structures.

\subsection{Density functional theory investigation of $\mathrm{N}$ doping}

The insight provided by acTEM into the atomic structure gives a convenient starting point to explore the energetics of the system by first principles calculations. A $6 \times 6$ supercell was used, containing 72 atoms for pristine graphene (hence, for the simulations incorporating $\mathrm{O}$ or $\mathrm{N}$, this corresponds to a doping of $\sim 1.4$ at $\%$, a similar concentration to the experimental situation above). Starting from previously published defect configurations [21,24], we optimized the atomic positions to find the ground state configurations. Here, the calculations analyze a free-standing graphene layer, whilst the experimental results are from graphene on copper; however, our previous experiments have shown that the graphene only weakly interacts with the copper surface and is well-described as a freestanding layer $[17,18]$. The calculations were performed to investigate the stability of structures with: $72 \mathrm{C}+$ $1 \mathrm{~N}$ (an adatom); $71 \mathrm{C}+1 \mathrm{~N}$ (substitution); $71 \mathrm{C}$ (monovacancy); $70 \mathrm{C}+1 \mathrm{~N}$ (monovacancy with $\mathrm{N}$ ); $70 \mathrm{C}$ (divacancy); and $69 \mathrm{C}+1 \mathrm{~N}$ (divacancy with $\mathrm{N}$ ). (a)

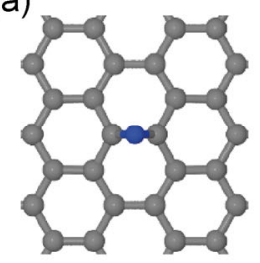

$\mathrm{N}$ adatom

(b)

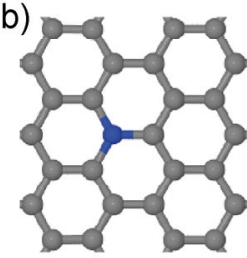

$\mathrm{N}$ substitution (c)

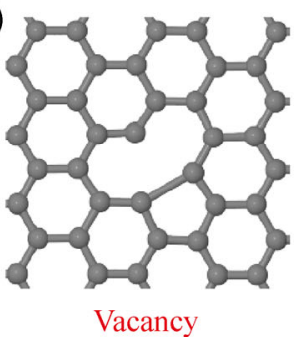

(d)

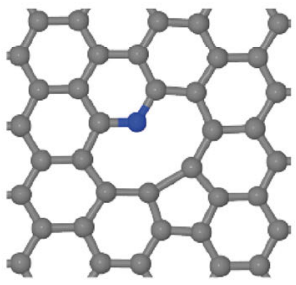

Vacancy + Pyridinic N (e)

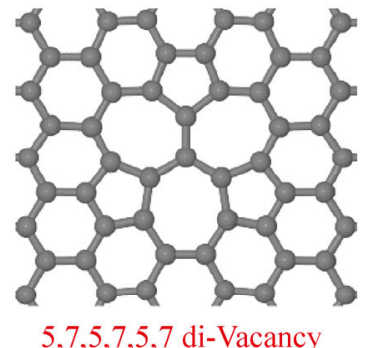

(f)

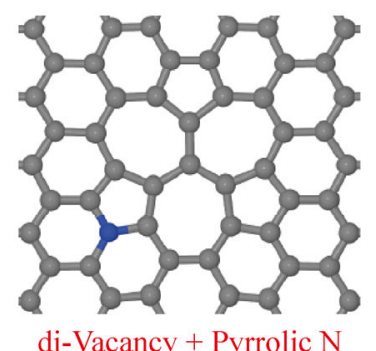

Figure 6 Energetically favorable structures, as predicted by density functional theory: (a) nitrogen adatom, (b) substitutional nitrogen, (c) monovacancy, (d) monovacancy with pyridinic nitrogen, (e) 5-7-5-7-5-7 divacancy, and (f) 5-7-5-7-5-7 divacancy with pyrrolic nitrogen. Carbon atoms are grey and nitrogen atoms are blue. 
For the divacancy, both the 5-8-5 and 5-7-5-7-5-7 reconstructions were investigated [24], but the latter was found to be more stable (consistent with previous reports $[25,28]$ and with the experimental results above); therefore, the results for the former have not been included here. The position of the nitrogen atom within the defects was varied; the most stable configurations are shown in Fig. 6.

As in the case of oxygen, the most stable position for the nitrogen adatom was found to be a bridge site. The formation energy indicates that it is stable with respect to atomic nitrogen, but unstable with respect to molecular nitrogen, see Table 1 . This is consistent with its formation under UHV, adding support to our assignment of the XPS peak at $396.6 \mathrm{eV}$ to the nitrogen adatom, and explains why it was readily removed by heating.

The lowest energy position of the nitrogen in the divacancy is a pyrrolic nitrogen, as shown in Fig. 6(f), with a formation energy of $+0.96 \mathrm{eV}$ relative to atomic nitrogen. Positioning the nitrogen instead on neighboring pyrrolic sites was only $\sim 0.5-0.7 \mathrm{eV}$ less energetically favorable. Nevertheless, the resultant structures and band structures were similar; for simplicity, we concentrate here only on the lowest energy structure. For the monovacancy, the most stable configuration (by $>2 \mathrm{eV}$ ) was found to be a pyridinic nitrogen with a formation energy consistent with previous reports $[29,30]$. The formation energies indicate that the pyridinic nitrogen stabilizes the monovacancy. The monovacancies in pristine graphene typically tend to be highly mobile under electron

Table 1 Formation energies of various defects derived from DFT calculations

\begin{tabular}{c|ccc}
\hline & \multicolumn{3}{|c}{$\begin{array}{c}\text { Formation energy relative } \\
\text { to graphene and }\end{array}$} \\
\cline { 2 - 4 } Defect & Stoichiometry & $\begin{array}{c}\text { Molecular } \\
\text { nitrogen } \\
\left(\mathrm{N}_{2}\right)(\mathrm{eV})\end{array}$ & $\begin{array}{c}\text { Atomic } \\
\text { nitrogen } \\
(\mathrm{N})(\mathrm{eV})\end{array}$ \\
\hline Nitrogen adatom & $72 \mathrm{C}, 1 \mathrm{~N}$ & 4.09 & -0.73 \\
Nitrogen substitution & $71 \mathrm{C}, 1 \mathrm{~N}$ & 0.49 & -4.33 \\
Monovacancy & $71 \mathrm{C}$ & 7.72 & 7.72 \\
$\begin{array}{c}\text { Monovacancy with } \\
\text { pyridinic nitrogen }\end{array}$ & $70 \mathrm{C}, 1 \mathrm{~N}$ & 5.23 & 0.42 \\
$7,5,7,5,7,5$ divacancy & $70 \mathrm{C}$ & 6.26 & 6.26 \\
$7,5,7,5,7,5$ divacancy & $69 \mathrm{C}, 1 \mathrm{~N}$ & 5.77 & 0.96 \\
with pyrrolic nitrogen & 6 & &
\end{tabular}

beam irradiation, rapidly migrating and merging to form the more stable divacancy [24, 31, 32]; however, here, we were able to acquire focal series, indicating that they did not diffuse rapidly and were stable. This supports our interpretation that the pyridinic nitrogen is associated with the vacancies.

In agreement with previous reports, we also found that adding further nitrogen to the monovacancy to form trimerized pyridine was energetically favorable [29]. However, for simplicity and because we have no direct experimental evidence as to the atomic locations of the nitrogen, we considered here only single nitrogen functionalities.

The formation energies listed in Table 1 do not give insight into the kinetics or formation mechanisms. Taken on their own, the formation energies would suggest that the introduction of nitrogen-containing defects in pristine graphene was not energetically favorable, whilst the incorporation of nitrogen into pre-existing vacancies was (although there is no indication that pre-existing vacancies are present in pristine graphene). However, previous DFT calculations have shown that vacancies and nitrogen dopants attract each other, i.e., that the presence of substitutional $\mathrm{N}$ increases the probability of the generation of vacancies and vice versa [30]. Furthermore, a previous study on the direct growth of N-doped CVD graphene also showed XPS evidence for pyridinic $\mathrm{N}$ that was presumed to be associated with the vacancies [21].

In summary, the use of atomic sources for low levels $(<5$ at $\%)$ of functionalization reversibly forms epoxy groups and irreversibly forms nitrogen containing groups, including substitutional nitrogen, and pyridinic and pyrrolic nitrogen associated with vacancies. The topological defects associated with the vacancies appear to be intrinsically linked to the nitrogen functionalization, and result in an irreversible damage to the hexagonal graphene lattice. This damage is also evident in the Raman spectra shown in the supporting information (Fig. S9).

\subsection{Electronic structure investigations}

The changes in the physical and chemical structure result in changes in the functional properties. We concentrate here on the changes in the electronic structure, which can be directly measured by ARPES; 

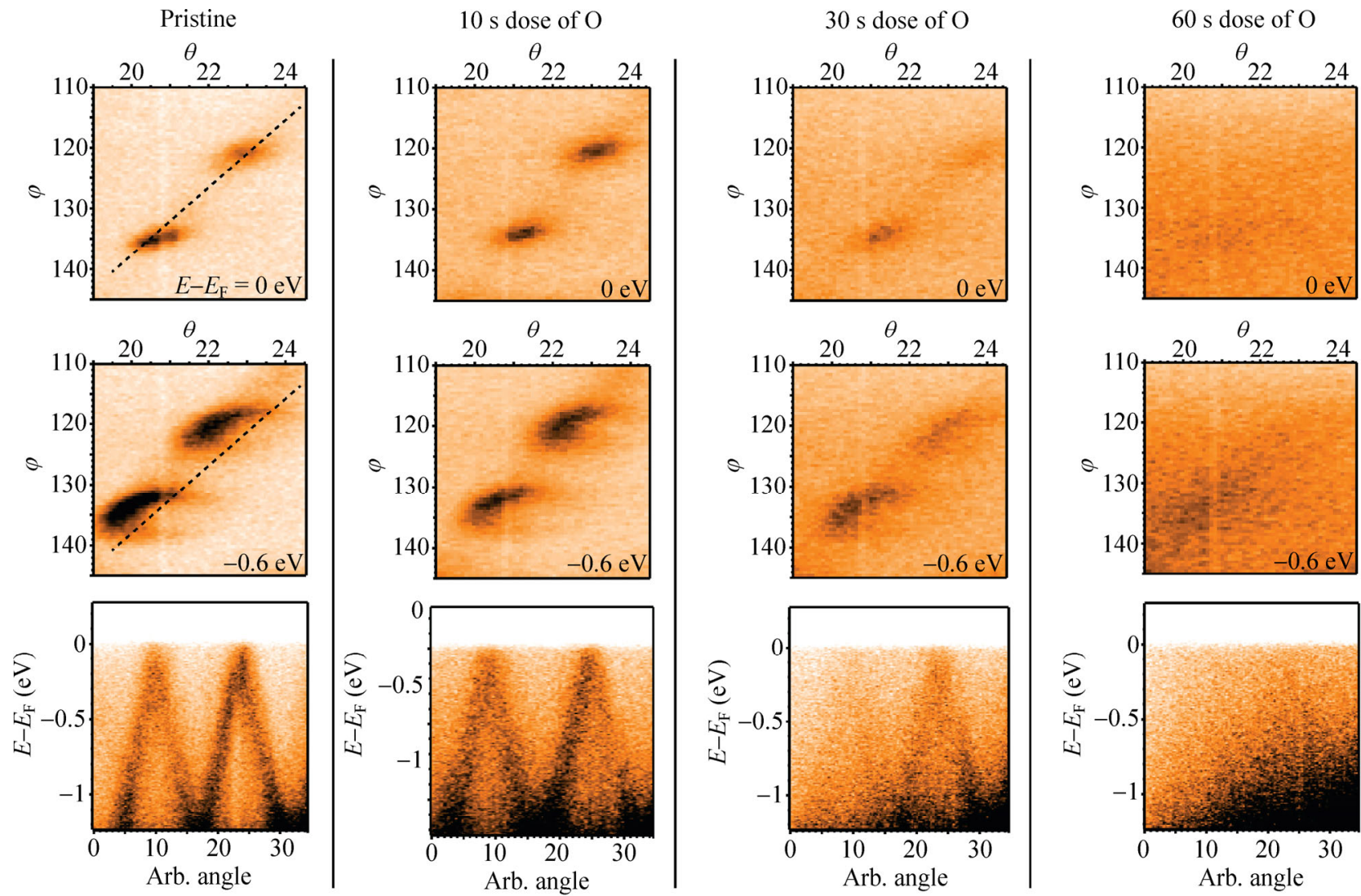

Figure 7 Angle resolved photoemission spectroscopy of graphene on copper before and after exposure to atomic oxygen with the exposure times as labeled. Top row: constant energy slice at the Fermi level; middle row: constant energy slice $0.6 \mathrm{eV}$ below the Fermi level; bottom row: slice through the Dirac cones perpendicular to the $\Gamma-\mathrm{K}$ direction.

this technique has been widely applied to study the electronic structure of graphene on various substrates, and has previously shown evidence for a metal insulator transition in graphene functionalized by atomic hydrogen [33]. The controlled functionalization using an atomic source and the high quality of graphene grown on copper allow us to investigate the changes in the electronic structure induced by the oxygen and nitrogen functionalization, and hence determine whether they dope graphene and whether electronic band gaps are induced around the Fermi level.

Figure 7 shows ARPES data before and after atomic oxygen doses. The ARPES data are three-dimensional, as they record the intensity as a function of the momenta in the in-plane $x$ and $y$ directions and as a function of the energy. Constant energy slices at the Fermi level (top), and $0.6 \mathrm{eV}$ below (middle), are shown along with slices perpendicular to the $\Gamma-\mathrm{K}$ direction (bottom). The two visible Dirac cones correspond to the two dominant orientations of graphene, as explained earlier in relation to Fig. 1. For clean graphene, the linear dispersion around the K-point is evident and the bands in each Dirac cone meet at the Fermi level. After exposure to atomic oxygen, the bands become more diffuse, but there is no evidence of shifting up or down in energy, i.e., there is no evidence that the atomic oxygen dopes graphene. After $60 \mathrm{~s}$, the band structure is no longer visible. Interestingly, the low energy electron diffraction of the sample at this stage still showed clear diffraction spots due to graphene, indicating that the structural order of the graphene lattice was retained, and that the broadening of the ARPES bands was not due to a roughened topography.

The data for clean graphene and after $10 \mathrm{~s}$ and $30 \mathrm{~s}$ exposures were fit to quantitatively extract the position of the Dirac point relative to the Fermi energy $\left(E_{\mathrm{D}}-E_{\mathrm{F}}\right.$, see Supporting Information Fig. S10 for details on the fitting process), and hence quantify the extent of the doping. Within experimental uncertainty, no doping or band gap was observed (see Table 2). 
Table 2 Effect of the exposure to atomic oxygen/nitrogen on the Dirac point energy

\begin{tabular}{ccccc}
\hline Dose & $\begin{array}{c}\text { Atomic } \\
\text { concentration }\end{array}$ & $\begin{array}{c}E_{\mathrm{D}}-E_{\mathrm{F}} \\
(\mathrm{meV})\end{array}$ & $\begin{array}{c}\text { Carrier } \\
\text { concentration } \\
\text { per unit cell } \\
(\mathrm{e})\end{array}$ & $\begin{array}{c}\text { Effective } \\
\text { charge per } \\
\mathrm{N}(\mathrm{e})\end{array}$ \\
\hline $\begin{array}{c}\text { Pristine } \\
\text { graphene }\end{array}$ & - & $0 \pm 10$ & - & - \\
$10 \mathrm{~s} \mathrm{O}$ & $0.7 \% \mathrm{O}$ & $0 \pm 10$ & - & - \\
$30 \mathrm{~s} \mathrm{O}$ & $3.1 \% \mathrm{O}$ & $0 \pm 10$ & - & - \\
$60 \mathrm{~s} \mathrm{O}$ & $5.0 \% \mathrm{O}$ & - & - & - \\
Pristine & - & $20 \pm 10$ & $2 \times 10^{-5} \pm$ & $1 \times 10^{-5}$ \\
graphene & & $-210 \pm$ & $-0.0022 \pm$ & $-0.07 \pm$ \\
$30 \mathrm{~s} \mathrm{~N}$ & $1.6 \% \mathrm{~N}$ & $-190 \pm$ & $-0.0018 \pm$ & $-0.04 \pm$ \\
& & 20 & 0.0004 & 0.01 \\
$60 \mathrm{~s} \mathrm{~N}$ & $2.6 \% \mathrm{~N}$ & -190.02 & - \\
$120 \mathrm{~s} \mathrm{~N}$ & $4.4 \% \mathrm{~N}$ & - & - & 0.0008 \\
\hline
\end{tabular}

Figure 8 shows the corresponding ARPES results for the atomic nitrogen exposure. Again, initially the band structure of graphene on copper is clear, and, within experimental uncertainty, there is no electronic doping. After $10 \mathrm{~s}$ of exposure to atomic nitrogen, the bands shifted down in energy and the bottom of the conduction band could be seen, indicating an n-type doping. As the exposure time increased, the bands became more diffuse, and after $60 \mathrm{~s}$, the band structure was no longer visible. At no stage was a band gap clearly visible, although the diffuse nature of the band structure after functionalization hampers a precise measurement. Thus, the atomic nitrogen exposure n-type dopes graphene, but, within the experimental uncertainty, no band gap is observed.

The shift in the Dirac point, $E_{\mathrm{D}}-E_{\mathrm{F}}$, is again extracted quantitatively by fitting the data, see Table 2 . The changes in the Dirac point are directly related to the changes in the carrier concentration, i.e., the electronic doping. If the Dirac point is shifted down in energy, then the carriers are electrons, if it is shifted up, they are holes. Close to the Dirac point, the band structure
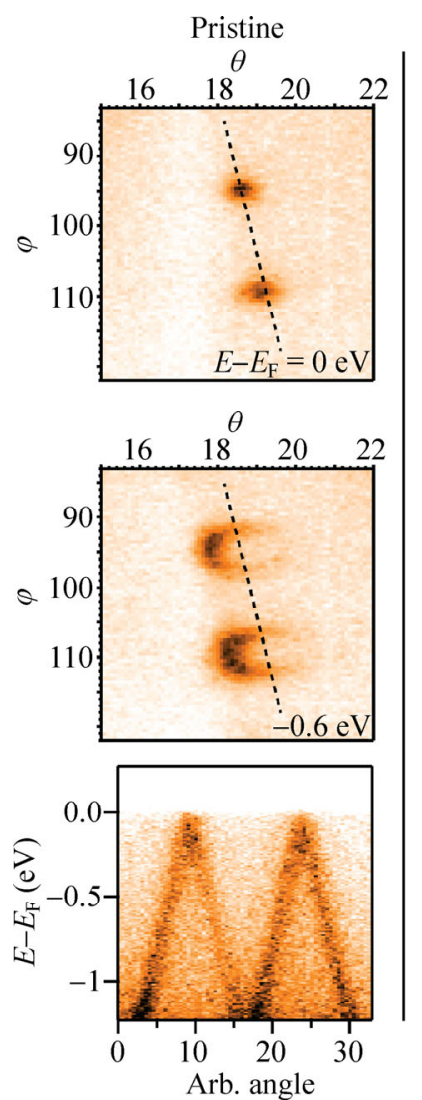
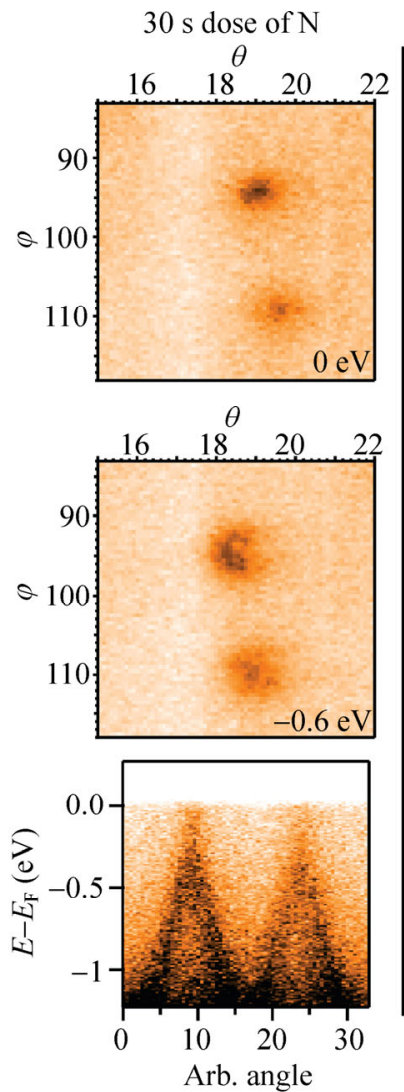
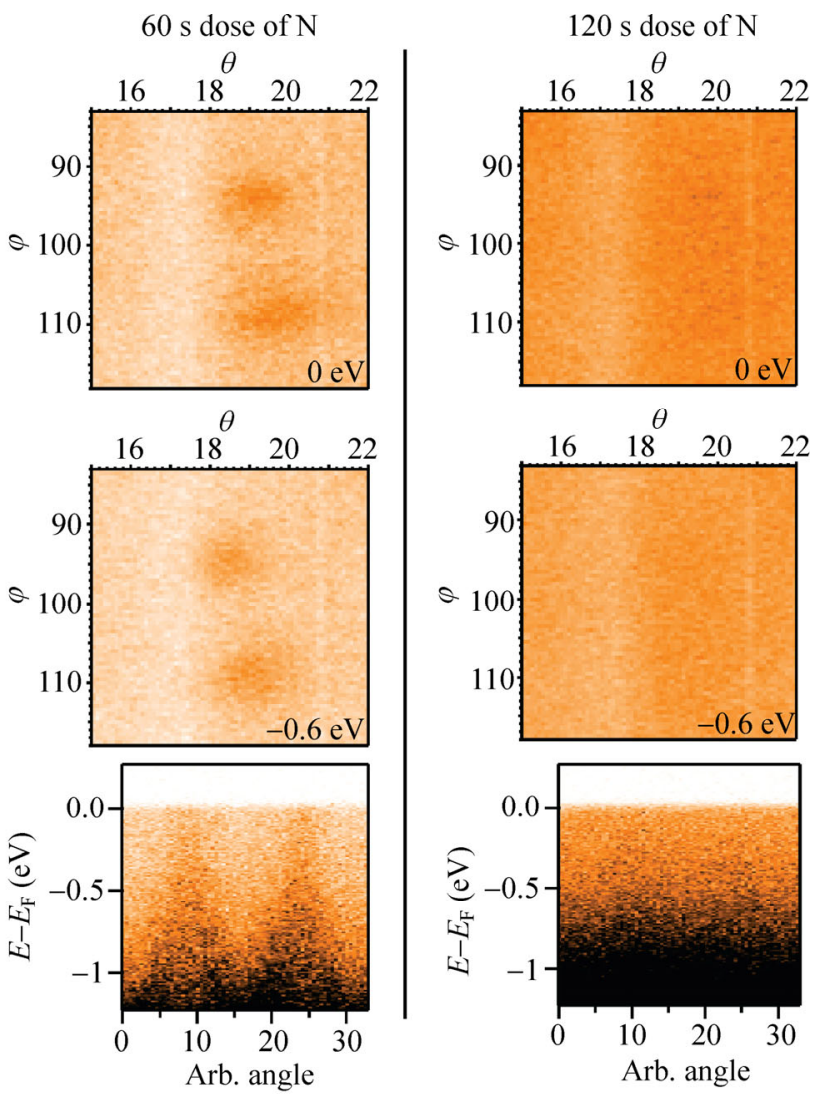

Figure 8 Angle resolved photoemission spectroscopy of graphene on copper before and after exposure to atomic nitrogen with the exposure times as labeled. Top row: constant energy slice at the Fermi level; middle row: constant energy slice $0.6 \mathrm{eV}$ below the Fermi level; bottom row: slice through the Dirac cones perpendicular to the $\Gamma-\mathrm{K}$ direction. 
of graphene can be accurately described by a simple tight-binding model. Calculating the density of states using the tight-binding model shows that the shift in energy of the Dirac point is directly related to the carrier concentration per unit cell by the equation $n=\frac{2}{\sqrt{3 \pi}}\left(\frac{E_{\mathrm{D}}-E_{\mathrm{F}}}{t}\right)^{2}$, where $t$ is the nearest neighbor hopping energy [34]. For graphene on copper, we have previously found that $t=2.7 \mathrm{eV}$ [17], which also fits well with the data here. Here, the changes in carrier concentration are due to the addition of nitrogen; the effective charge added to the graphene carrier concentration per $N$ atom can be calculated from the carrier concentration divided by the average concentration of $N$ atoms. Table 2 shows the carrier concentration per unit cell and the effective charge contributed to the carrier concentration per $N$ atom, calculated from the Dirac point shift using the equation above.

The disappearance of the graphene band structure after $60 \mathrm{~s}$ of exposure, for both atomic oxygen and nitrogen exposure, is consistent with the disorder induced localization of the electronic states, as previously observed for the exposure of graphene on $\mathrm{SiC}$ to atomic hydrogen [33]. As described by Bostwick et al. [33], the disorder induced by the functionalization can cause a metal-insulator transition because of a strong Anderson localization. An ab initio study of charge transport through ozone-functionalized graphene similarly revealed a metal-insulator transition through varying the coverage of the epoxide defects from $0.1 \%$ to $4 \%$ [12]. Thus, our results suggest that the dominant electronic effect of the disordered functionalization is to convert graphene from a semimetal to an electronically disordered, localized system. For charge transport, this would imply a dramatic decrease in the mobility and a transition to hopping mediated transport, as has been observed for reduced graphene oxide [35].

Understanding the doping effect of atomic nitrogen requires the consideration of all the types of functional groups that it creates. As a first approximation, we may expect that nitrogen will act as an electron donor in graphene, in which case the level of doping would be directly correlated to the atomic percentage of nitrogen present. However, this is too simplistic. To gain further insight, DFT was used to investigate the doping due to the defect structures shown in Fig. 6, as well as the epoxy group. The doping was calculated from the electronic band structure of the supercell by measuring the shift in the Dirac point (see Methods and Supporting Information for details, including band structure results). Table 3 shows the calculated shift in the Dirac point for the defects, along with the effective contribution to the carrier concentration per defect, calculated from the Dirac point shift as described above.

For the oxygen adatom (epoxy), only negligible doping is predicted, consistent with the experimental results. Among the nitrogen containing groups, only the nitrogen substitution is found to n-type dope graphene, with an effective charge carrier contribution per defect of $-0.28 \mathrm{e}$. The nitrogen adatom gives negligible doping, similar to the epoxy group, whilst the pyridinic and pyrrolic nitrogen, owing to their association with the vacancy structures, are predicted to p-type dope graphene in agreement with a previous DFT study [36]. Again, this can be understood at a simplistic level: the vacancy equates to a missing electron and hence a p-type doping effect. These oversimplifications must be treated with caution as the monovacancy with pyridinic nitrogen considerably contributes to higher levels of p-type doping than the monovacancy on its own.

The overall effect of the functionalization on the carrier concentration is due to the combination of the present functionalities. The balance between the

Table 3 Dirac point shifts and effective charge contributions derived from DFT calculations using a $6 \times 6$ supercell $(72$ carbon atoms for pristine graphene including $1 \mathrm{O}$ or $\mathrm{N}$ atom, corresponding to a doping concentration of $\sim 1.4$ at.\%)

\begin{tabular}{ccc}
\hline Defect & $E_{\mathrm{D}}-E_{\mathrm{F}}(\mathrm{eV})$ & $\begin{array}{c}\text { Effective charge } \\
\text { per defect }(\mathrm{e})\end{array}$ \\
\hline Oxygen adatom (epoxy) & 0.015 & $0.000,4$ \\
Nitrogen adatom & 0.090 & 0.015 \\
Nitrogen substitution & -0.390 & -0.28 \\
Monovacancy & 0.060 & 0.007 \\
$\begin{array}{c}\text { Monovacancy with } \\
\text { pyridinic nitrogen }\end{array}$ & 0.330 & 0.19 \\
$\begin{array}{c}\text { 7, 5, 7, 5, 7, 5 divacancy } \\
\text { 7, 5, 7, 5, 7, 5 divacancy } \\
\text { with pyrrolic nitrogen }\end{array}$ & 0.750 & 1.0 \\
\hline
\end{tabular}


n-doping substitution and p-doping pyridinic/pyrrolic nitrogen experimentally results in a low level of n-type doping. This explains why the experimentally observed carrier concentrations per nitrogen atom are so low, considerably less than those expected for purely substitutional nitrogen. Simple considerations on the relative abundance of the substitutional:pyridinic/ pyrrolic nitrogen (ratio from the XPS above) would suggest that, after atomic nitrogen exposure, graphene should be slightly p-doped (see Supporting Information Fig. S13). However, as discussed above, considering the formation energies, it is more favorable for multiple $\mathrm{N}$ atoms to be associated with a single vacancy. In that case, the effective charge per $\mathrm{N}$ atom (p-doping) is strongly reduced [29]. Hence, the experimental observation that overall graphene is n-doped, combined with the relative abundances calculated from XPS, implies that multiple nitrogen atoms are associated with the vacancies, and that the extended topological defects play an important role in determining the doping.

Thus, the electronic structure investigations show that, at functionalization levels of the order a few at $\%$, the dominant effect is a metal-insulator transition due to the localization caused by strong scattering from the defects, as previously found for hydrogenated graphene [33]. We expect this to be a generic effect for all forms of disordered functionalization.

The oxygen functionalization, which at this level does not create vacancies, does not dope graphene. The nitrogen functionalization is more complicated because of the range of functional groups induced; the competition between $n$-doping substitutional nitrogen and p-doping pyridinic and pyrrolic nitrogen means that overall only a weak n-doping is observed. Interestingly, a recent investigation into nitrogen ion implantation of graphene at high levels of nitrogen implantation up to $\sim 20$ at $\%$ showed that the ratio of substitutional to pyridinic/pyrrolic could be increased by annealing [23]. This result was also observed at low nitrogen concentrations by Usachov et al. [21,37], who found significant $n$-doping at low levels of nitrogen (0.4 at $\%)$, which can be explained by the high fraction of substitutional nitrogen in that case.

For the disordered functionalization studied here, we were not able to distinguish the formation of a band gap, although the broadening of the band structure (reflective of the localization and the heterogeneous nature of the functionalization) limits the accuracy with which this can be determined. This is in contrast to the prior ARPES work by Usachov et al. on nitrogen doped graphene, in which a small band gap of $\sim 0.2 \mathrm{eV}$ at a very low nitrogen concentration $(\sim 0.4$ at $\%)$ was observed [21].

\section{Conclusions}

The work presented here demonstrates the very significant changes that low levels of covalent functionalization (up to 5 at\%) make to the physical, chemical, and electronic structure of graphene. Clearly, the properties of functionalized graphene are very distinct from those of pristine graphene: functionalized graphenes are, in effect, a different class of materials. It is also clear that functionalization with different species, even at the same level and under similar conditions, can result in radically different physical structures.

An important role is played by the vacancies and the resultant topological defects in the graphene lattice. The atomic nitrogen functionalization is shown to introduce structures with associated pyridinic and pyrrolic nitrogen in an irreversible process. At low levels, atomic oxygen does not introduce vacancies and its effects are readily reversible. The vacancies and topological defects are more reactive; hence, the incorporation of nitrogen and the high electrocatalytic activity of $\mathrm{N}$-doped graphene are observed. There appears to be a tendency for the vacancies to cluster, creating extended topological defects. The epoxide groups formed by atomic oxygen do not electronically dope graphene, whilst the competition between the $\mathrm{n}$-doping substitutional nitrogen and $\mathrm{p}$-doping pyridinic and pyrrolic nitrogen results in a weak net $n$-doping of graphene.

However, for disordered covalent functionalization, the dominant electronic effect appears to be the localization resulting in a metal-insulator transition. This will result in the evolution of the transport properties from the semi-metallic behavior of graphene to a hopping mediated transport (like reduced graphene oxide), to an insulating material. As almost all the covalent functionalization of graphene is disordered, 
with notable exceptions such as fluorographene [38] and graphane [39], caution is necessary in the application of the covalent functionalization as a route for the band engineering of graphene for electronic applications.

\section{Methods}

Growth. Graphene was grown via LP-CVD on low cost copper foils $(99.5 \%$ purity, $0.025 \mathrm{~mm}$ thick, Alfa Aesar product number 46365). The foils were cleaned with a $10 \mathrm{~s}$ electropolish at $5 \mathrm{~V}$ with $1.5 \mathrm{~A}$ to remove the surface contamination. This was followed by rinsing in acetone and then isopropanol, before drying in nitrogen. The foils were then placed in a 1 in quartz work tube that was pumped to below $10^{-3}$ mbar. The sample was heated to $1,000{ }^{\circ} \mathrm{C}$ under 20 standard cubic centimeters per minute $(\mathrm{sccm})$ hydrogen (pressure $10^{-1}$ mbar). The hydrogen flow was sustained throughout the growth process. After annealing at $1,000{ }^{\circ} \mathrm{C}$ for $20 \mathrm{~min}, 10 \mathrm{sccm}$ methane was introduced for $10 \mathrm{~min}$. The methane flow rate was dropped to $2 \mathrm{sccm}$ while the sample was cooled to $600{ }^{\circ} \mathrm{C}$, and then stopped while cooling to room temperature.

Functionalization. Graphene on $\mathrm{Cu}$ foil was exposed to controlled doses of atomic $\mathrm{O}$ and atomic $\mathrm{N}$ in UHV using a Gen 2 microwave plasma source (Tectra $\mathrm{GmbH}$ ) in atom mode (remote from the plasma with the ions filtered out); the doses were fixed by operating at constant power and gas pressure with varying exposure time.

Photoemission spectroscopy. ARPES and XPS measurements were taken at the Antares beam line at Synchrotron Soleil using a Scienta R4000 2D detector [40]. For all the measurements, the samples were cooled to a temperature $<200 \mathrm{~K}$. For the XPS measurements, a photon energy of $700 \mathrm{eV}$ was used, while for ARPES measurements, an energy of $100 \mathrm{eV}$ was used. The binding energy scale was calibrated using the Fermi edge visible from copper.

TEM analysis. Graphene was transferred from the copper foil to Quantifoil TEM grids with equally spaced $2 \mu \mathrm{m}$ holes for the TEM measurements. Graphene on copper was coated with poly(methyl methacrylate)
(PMMA) to provide support while the copper was removed with ammonium persulfate. The graphene/ PMMA stack was cleaned in de-ionised water and then scooped onto the grids. After drying, the PMMA was removed with acetone, and heated under hydrogen at low pressure $\left(20 \mathrm{sccm} \mathrm{H}_{2}, 10^{-1}\right.$ mbar, $3 \mathrm{~h}$ ). For the $\mathrm{N}$ - and O-dosed experiments, graphene covered TEM grids were transferred in UHV in copper enclosures for dosing. The TEM measurements were taken with a JEOL ARM 200F TEM instrument with double aberration correction operating at $80 \mathrm{kV}$. The images were recorded on a Gatan SC-1000 Orius chargecoupled device (CCD), with typical exposures of $1 \mathrm{~s}$. For the exit wave reconstruction, a custom plug-in for Digital Micrograph recorded 20 images around focus with focal increments of $1.4 \mathrm{~nm}$ and $1 \mathrm{~s}$ exposure. These images are then combined using the focal and tilt series reconstruction (FTSR) to find the phase of the wave function just after passing through the sample [41].

DFT and image simulation. DFT calculations were performed with the CASTEP plane wave density functional code using generalized gradient approximation (GGA) exchange-correlation functionals in the PW91 formulations and ultrasoft pseudopotentials created on the fly using the standard pseudo-atom definitions distributed with the academic release of CASTEP 6.1 [42-44]. The defects were embedded in a hexagonal $6 \times 6$ supercell of graphene with a $28 \AA$ vacuum gap in the orthogonal direction. We sampled the reciprocal space on a $2 \times 2 \times 1$ Monkhorst-Pack grid [45] and used a plane wave cutoff energy of $560 \mathrm{eV}$ (620 eV for systems containing oxygen). At this level of representation, energies were converged to $10 \mathrm{meV} /$ atom and atomic forces to within $0.002 \mathrm{eV} / \AA$. Starting from published defect structures [21, 24, 25], the positions of the atoms were optimized using a variation of the Broyden-Fletcher-Goldfarb-Shanno method (BFGS method) [46] until a force tolerance of $0.015 \mathrm{eV} / \AA$ was achieved. The structures were visualized using Jmol, an open-source Java viewer for chemical structures in 3D (http://www.jmol.org/). The effective band structures were derived following the method described by Popescu and Zunger [47, 48] using bs_sc2pc [49], a recently implemented add-on released with the academic release of CASTEP 7.0. 
The TEM images were simulated from the calculated DFT potentials using standard multislice procedures.

\section{Acknowledgements}

EPSRC is thanked for support through studentships to AJM, MAD and JJM and through Grants (Nos.EP/ K005200/1 and EP/H00341X/1. This work made use of the facilities of HECToR, the UK's national highperformance computing service, which was provided by UoE HPCx Ltd at the University of Edinburgh, Cray Inc and NAG Ltd, and funded by the Office of Science and Technology through EPSRC's High End Computing Programme. Additional computational resources were provided by MidPlus (EPSRC Grant No. EP/K000233/1). We acknowledge support via the CALIPSO TransNational Access Program from the European Community's Seventh Framework Programme (FP7/2007-2013) under grant agreement $n^{\circ}$ 312284. We thank J. Rourke for helpful discussions.

Electronic Supplementary Material: Supplementary material with further experimental details, photoemission data and analysis, Raman spectroscopy, and DFT is available in the online version of this article at http://dx.doi.org/10.1007/s12274-015-0768-0.

Open Access: This article is distributed under the terms of the Creative Commons Attribution License which permits any use, distribution, and reproduction in any medium, provided the original author(s) and the source are credited.

\section{References}

[1] Dreyer, D. R.; Park, S.; Bielawski, C. W.; Ruoff, R. S. The chemistry of graphene oxide. Chem. Soc. Rev. 2010, 39, 228-240.

[2] Liu, N.; Luo, F.; Wu, H. X.; Liu, Y. H.; Zhang, C.; Chen, J. One-step ionic-liquid-assisted electrochemical synthesis of ionic-liquid-functionalized graphene sheets directly from graphite. Adv. Funct. Mater. 2008, 18, 1518-1525.

[3] Dreyer, D. R.; Todd, A. D.; Bielawski, C. W. Harnessing the chemistry of graphene oxide. Chem. Soc. Rev. 2014, 43, 5288-5301.

[4] Wang, Y.; Shao, Y. Y.; Matson, D. W.; Li, J. H.; Lin, Y. H.
Nitrogen-doped graphene and its application in electrochemical biosensing. ACS Nano 2010, 4, 1790-1798.

[5] Mo, Z. Y.; Zheng, R. P.; Peng, H. L.; Liang, H. G.; Liao, S. J. Nitrogen-doped graphene prepared by a transfer doping approach for the oxygen reduction reaction application. $J$. Power Sources 2014, 245, 801-807.

[6] Wood, K. N.; O'Hayre, R.; Pylypenko, S. Recent progress on nitrogen/carbon structures designed for use in energy and sustainability applications. Energy Environ. Sci. 2014, 7, 1212-1249.

[7] Wang, H. B.; Maiyalagan, T.; Wang, X. Review on recent progress in nitrogen-doped graphene: Synthesis, characterization, and its potential applications. ACS Catal. 2012, 2, 781-794.

[8] Qu, L. T.; Liu, Y.; Baek, J. B.; Dai, L. M. Nitrogen-doped graphene as efficient metal-free electrocatalyst for oxygen reduction in fuel cells. ACS Nano 2010, 4, 1321-1326.

[9] Wei, D. C.; Liu, Y. Q.; Wang, Y.; Zhang, H. L.; Huang, L. P.; $\mathrm{Yu}, \mathrm{G}$. Synthesis of $\mathrm{N}$-doped graphene by chemical vapor deposition and its electrical properties. Nano Lett. 2009, 9, 1752-1758.

[10] Zabet-Khosousi, A.; Zhao, L. Y.; Pálová, L.; Hybertsen, M. S.; Reichman, D. R.; Pasupathy, A. N.; Flynn, G. W. Segregation of sublattice domains in nitrogen-doped graphene. J. Am. Chem. Soc. 2014, 136, 1391-1397.

[11] Yuan, J. T.; Ma, L. P.; Pei, S. F.; Du, J. H.; Su, Y.; Ren, W. C.; Cheng, H. M. Tuning the electrical and optical properties of graphene by ozone treatment for patterning monolithic transparent electrodes. ACS Nano 2013, 7, 4233-4241.

[12] Leconte, N.; Moser, J.; Ordejón, P.; Tao, H. H.; Lherbier, A.; Bachtold, A.; Alsina, F.; Sotomayor Torres, C. M.; Charlier, J. C.; Roche, S. Damaging graphene with ozone treatment: A chemically tunable metal-insulator transition. ACS Nano 2010, 4, 4033-4038.

[13] Peltekis, N.; Kumar, S.; McEvoy, N.; Lee, K.; Weidlich, A.; Duesberg, G. S. The effect of downstream plasma treatments on graphene surfaces. Carbon 2012, 50, 395-403.

[14] Hossain, M. Z.; Johns, J. E.; Bevan, K. H.; Karmel, H. J.; Liang, Y. T.; Yoshimoto, S.; Mukai, K.; Koitaya, T.; Yoshinobu, J.; Kawai, M. et al. Chemically homogeneous and thermally reversible oxidation of epitaxial graphene. Nat. Chem. 2012, 4, 305-309.

[15] Barinov, A.; Malcioğlu, B. O.; Fabris, S.; Sun, T.; Gregoratti, L.; Dalmiglio, M.; Kiskinova, M. Initial stages of oxidation on graphitic surfaces: Photoemission study and density functional theory calculations. J. Phys. Chem. C 2009, 113, 9009-9013.

[16] Marsden, A. J.; Phillips, M.; Wilson, N. R. Friction force microscopy: A simple technique for identifying graphene on 
rough substrates and mapping the orientation of graphene grains on copper. Nanotechnology 2013, 24, 255704.

[17] Wilson, N. R.; Marsden, A. J.; Saghir, M.; Bromley, C. J.; Schaub, R.; Costantini, G.; White, T. W.; Partridge, C.; Barinov, A.; Dudin, P. et al. Weak mismatch epitaxy and structural feedback in graphene growth on copper foil. Nano Res. 2013, 6, 99-112.

[18] Marsden, A. J.; Asensio, M. C.; Avila, J.; Dudin, P.; Barinov, A.; Moras, P.; Sheverdyaeva, P. M.; White, T. W.; Maskery, I.; Costantini, G. et al. Is graphene on copper doped? Phys. Status Solidi-Rapid Res. Lett. 2013, 7, 643-646.

[19] Blume, R.; Kidambi, P. R.; Bayer, B. C.; Weatherup, R. S.; Wang, Z. J.; Weinberg, G.; Willinger, M. G.; Greiner, M.; Hofmann, S.; Knop-Gericke, A. et al. The influence of intercalated oxygen on the properties of graphene on polycrystalline $\mathrm{Cu}$ under various environmental conditions. Phys. Chem. Chem. Phys. 2014, 16, 25989-26003.

[20] Sheng, Z. H.; Shao, L.; Chen, J. J.; Bao, W. J.; Wang, F. B.; Xia, X. H. Catalyst-free synthesis of nitrogen-doped graphene via thermal annealing graphite oxide with melamine and its excellent electrocatalysis. ACS Nano 2011, 5, 4350-4358.

[21] Usachov, D.; Vilkov, O.; Grüneis, A.; Haberer, D.; Fedorov, A.; Adamchuk, V. K.; Preobrajenski, A. B.; Dudin, P.; Barinov, A.; Oehzelt, M. et al. Nitrogen-doped graphene: Efficient growth, structure, and electronic properties. Nano Lett. 2011, 11, 5401-5407.

[22] Zhang, L.; Ye, Y. F.; Cheng, D. D.; Zhang, W. H.; Pan, H. B.; Zhu, J. F. Simultaneous reduction and N-doping of graphene oxides by low-energy $\mathrm{N}_{2}{ }^{+}$ion sputtering. Carbon 2013, 62, 365-373.

[23] Scardamaglia, M.; Aleman, B.; Amati, M.; Ewels, C.; Pochet, P.; Reckinger, N.; Colomer, J. F.; Skaltsas, T.; Tagmatarchis, N.; Snyders, R. et al. Nitrogen implantation of suspended graphene flakes: Annealing effects and selectivity of $\mathrm{sp}^{2}$ nitrogen species. Carbon 2014, 73, 371-381.

[24] Banhart, F.; Kotakoski, J.; Krasheninnikov, A. V. Structural defects in graphene. ACS Nano 2011, 5, 26-41.

[25] Terrones, H.; Lv, R. T.; Terrones, M.; Dresselhaus, M. S. The role of defects and doping in 2D graphene sheets and 1D nanoribbons. Rep. Prog. Phys. 2012, 75, 062501.

[26] Gómez-Navarro, C.; Meyer, J. C.; Sundaram, R. S.; Chuvilin, A.; Kurasch, S.; Burghard, M.; Kern, K.; Kaiser, U. Atomic structure of reduced graphene oxide. Nano Lett. 2010, 10, 1144-1148.

[27] Cretu, O.; Krasheninnikov, A. V.; Rodríguez-Manzo, J. A.; Sun, L. T.; Nieminen, R. M.; Banhart, F. Migration and localization of metal atoms on strained graphene. Phys. Rev. Lett. 2010, 105, 196102.
[28] Lee, G. D.; Wang, C. Z.; Yoon, E.; Hwang, N. M.; Kim, D. Y.; Ho, K. M. Diffusion, coalescence, and reconstruction of vacancy defects in graphene layers. Phys. Rev. Lett. 2005, 95, 205501.

[29] Fujimoto, Y.; Saito, S. Formation, stabilities, and electronic properties of nitrogen defects in graphene. Phys. Rev. B 2011, 84, 245446

[30] Hou, Z. F.; Wang, X. L.; Ikeda, T.; Terakura, K.; Oshima, M.; Kakimoto, M.; Miyata, S. Interplay between nitrogen dopants and native point defects in graphene. Phys. Rev. B 2012, 85, 165439.

[31] El-Barbary, A. A.; Telling, R. H.; Ewels, C. P.; Heggie, M. I.; Briddon, P. R. Structure and energetics of the vacancy in graphite. Phys. Rev. B 2003, 68, 144107.

[32] Robertson, A. W.; Allen, C. S.; Wu, Y. M. A.; He, K.; Olivier, J.; Neethling, J.; Kirkland, A. I.; Warner, J. H. Spatial control of defect creation in graphene at the nanoscale. Nat. Commun. 2012, 3, 1144.

[33] Bostwick, A.; McChesney, J.; Emtsev, K.; Seyller, T.; Horn, K.; Kevan, S.; Rotenberg, E. Quasiparticle transformation during a metal-insulator transition in graphene. Phys. Rev. Lett. 2009, 103, 056404.

[34] Castro Neto, A. H.; Guinea, F.; Peres, N. M. R.; Novoselov, K. S.; Geim, A. K. The electronic properties of graphene. Rev. Mod. Phys. 2009, 81, 109-162.

[35] Gómez-Navarro, C.; Weitz, R. T.; Bittner, A. M.; Scolari, M.; Mews, A.; Burghard, M.; Kern, K. Electronic transport properties of individual chemically reduced graphene oxide sheets. Nano Lett. 2007, 7, 3499-3503.

[36] Hou, Z. F.; Wang, X. L.; Ikeda, T.; Terakura, K.; Oshima, M.; Kakimoto, M. Electronic structure of N-doped graphene with native point defects. Phys. Rev. B 2013, 87, 165401.

[37] Usachov, D.; Fedorov, A.; Vilkov, O.; Senkovskiy, B.; Adamchuk, V. K.; Yashina, L. V.; Volykhov, A. A.; Farjam, M.; Verbitskiy, N. I.; Grüneis, A. et al. The chemistry of imperfections in N-graphene. Nano Lett. 2014, 14, 48924988.

[38] Nair, R. R.; Ren, W. C.; Jalil, R.; Riaz, I.; Kravets, V. G.; Britnell, L.; Blake, P.; Schedin, F.; Mayorov, A. S.; Yuan, S. J. et al. Fluorographene: A two-dimensional counterpart of Teflon. Small 2010, 6, 2877-2884.

[39] Elias, D. C.; Nair, R. R.; Mohiuddin, T. M. G.; Morozov, S. V.; Blake, P.; Halsall, M. P.; Ferrari, A. C.; Boukhvalov, D. W.; Katsnelson, M. I.; Geim, A. K. et al. Control of graphene's properties by reversible hydrogenation: Evidence for graphane. Science 2009, 323, 610-613.

[40] Avila, J.; Razado-Colambo, I.; Lorcy, S.; Lagarde, B.; Giorgetta, J. L.; Polack, F.; Asensio, M. C. ANTARES, a 
scanning photoemission microscopy beamline at SOLEIL. $J$ Phys. Conf. Ser. 2013, 425, 192023.

[41] Meyer, R. R.; Kirkland, A. I.; Saxton, W. O. A new method for the determination of the wave aberration function for high resolution TEM: 1. Measurement of the symmetric aberrations. Ultramicroscopy 2002, 92, 89-109.

[42] Clark, S. J.; Segall, M. D.; Pickard, C. J.; Hasnip, P. J.; Probert, M. I. J.; Refson, K.; Payne, M. C. First principles methods using CASTEP. Z. Kristall. 2005, 220, 567-570.

[43] Perdew, J. P.; Wang, Y. Accurate and simple analytic representation of the electron-gas correlation energy. Phys. Rev. B 1992, 45, 13244-13249.

[44] Vanderbilt, D. Soft self-consistent pseudopotentials in a generalized eigenvalue formalism. Phys. Rev. B 1990, 41,
7892-7895.

[45] Monkhorst, H. J.; Pack, J. D. Special points for Brillouinzone integrations. Phys. Rev. B 1976, 13, 5188-5192.

[46] Byrd, R. H.; Nocedal, J.; Schnabel, R. B. Representations of quasi-Newton matrices and their use in limited memory methods. Math. Program. 1994, 63, 129-156.

[47] Popescu, V.; Zunger, A. Effective band structure of random alloys. Phys. Rev. Lett. 2010, 104, 236403.

[48] Popescu, V.; Zunger, A. Extracting E versus $\overrightarrow{\boldsymbol{k}}$ effective band structure from supercell calculations on alloys and impurities. Phys. Rev. B 2012, 85, 085201.

[49] Brommer, P.; Quigley, D. Automated effective band structures for defective and mismatched supercells. J. Phys. Condens. Matter 2014, 26, 485501. 